Reprod. Nutr. Dévelop., 1984, 24 (2), 179-187.

\title{
Micro-environnement thymique et différenciation des lymphocytes T
}

\author{
Martine PAPIERNIK
}

INSERM U 25, Hópital Necker,

161, rue de Sèvres, 75730 Paris Cedex 15.

Summary. The thymic micro-environment and $T$ lymphocyte differentiation.

T cell precursors, which are derived from fœtal liver and later from bone marrow, are obliged to colonize the thymus to differentiate into mature $T$ cells. Multiple factors, secreted by cells of different types and origins, are involved in the constitution of a suitable micro-environment. Thymic epithelial cells have an endo-ectodermal origin, while the epithelial rudiment is secondarily colonized by mesodermal precursors of lymphocytes, macrophages and interdigitating cells.

The migration of stem cells into the thymus is controlled by chemotactic factors which are derived from epithelial cells as well as from mature thymic medularry cells.

Intra-thymic differentiation is controlled by thymic humoral factors secreted by epithelial cells. One of these factors, the "facteur thymique sérique " (FTS) (now called thymulin), has been isolated from the serum and also plays a role in the later extra-thymic maturation process. Thymic hormones are able to generate differentiation antigens on the surface of pre-thymic precursors. They have complex effects on the immunological function of post-thymic precursors. In addition to thymic hormones, other signals, such as interleukins, are necessary. Dendritic-shaped phagocytic cells of the thiymic reticulum are able to secrete $\mathrm{IL}_{1}$ which is necessary for the secretion of $\mathrm{IL}_{2}$ by mature thymic medullary lymphocytes.

MHC antigens on thymic epithelial cells and other factors, such as prostaglandins, also play important roles in intra-thymic $T$ cell differentiation.

Le thymus contrôle la différenciation des lymphocytes $T$, et l'on sait depuis les expériences de Miller (Miller, 1962) que la thymectomie néonatale chez la souris entraîne l'absence de lymphocytes $T$ matures dans les organes périphériques. Depuis vingt ans, et cette première découverte, une quantité considérable de travaux ont tenté d'analyser précisément le rôle du thymus dans cette différenciation des lymphocytes T. Il a été montré, notamment par les travaux de Stutman (1978), que les cellules souches ont l'obligation de séjourner dans le thymus pour accomplir leur cycle complet de maturation. A l'intérieur du thymus, les cellules souches baignent dans un micro-environnement complexe oú interviennent des cellules de type et d'origine embryologique différents. Parmi celles-ci, les cellules épithéliales ont été les plus étudiées car elles sont à l'origine de la sécrétion des hormones thymiques (Bach et Carnaud, 1976). Pourtant, d'autres cellules jouent un rôle dans la constitution du micro-environnement thymique, par exemple, par la sécré- 
tion de facteurs chimiotactiques nécessaires à la migration des cellules souches vers le thymus, ou par la sécrétion d'interleukines. Les interleukines peuvent intervenir comme des amplificateurs des signaux de maturation produits par les cellules épithéliales. La différenciation des lymphocytes $T$ est donc un phénomène complexe dans lequel interviennent des facteurs multiples dont les inter-relations et la place dans la cascade des événements restent encore à analyser.

\section{Les éléments cellulaires constitutifs du micro-environnement thymique.}

L'ébauche initiale du thymus a une origine endo-ectodermique et est constituée de cellules épithéliales. Cette ébauche épithéliale va être colonisée secondairement par des cellules venues du foie fœetal, puis de la moelle osseuse, et qui vont donner naissance, d'une part, aux lymphocytes et, d'autre part, aux macrophages et aux cellules interdigitées qui leur sont proches. Lymphocytes, cellules interdigitées et macrophages ont donc une origine embryologique différente des cellules épithéliales puisqu'elles proviennent du feuillet mésodermique. Cette colonisation de l'ébauche par des cellules extrathymiques se fait chez le souris entre le $7^{\mathrm{e}}$ et le $9^{\mathrm{e}}$ jour de gestation.

Les premiers lymphocytes qui apparaissent dans le thymus sont des cellules de type mature, localisées à la zone médullaire (Papiernik, 1970 ; Ceredig, Mac Donalld et Jenkinson, 1983). Peu de temps après se différencient les lymphocytes corticaux fonctionnellement immatures. Chez l'adulte, la population de thymocytes matures constitue 5 à $10 \%$ de l'ensemble des thymocytes, les lymphocytes corticaux constituent 90 à $95 \%$ des cellules.

\section{Les facteurs de la migration intra-thymique.}

Les cellules souches des lymphocytes $T$ vont pénétrer dans l'ébauche thymique, sans que l'on sache pour le moment si ce sont des cellules neutres, ou des cellules déjà engagées dans le processus de filiation vers une lignée $T$. Le processus de migration a été bien étudié chez les oiseaux par l'équipe de Le Douarin (Jotereau et al., 1980). Ces travaux ont montré que les cellules souches sont attirées par le thymus si celui-ci est dans un stade de réceptivité. Cet état de réceptivité est conditionné par l'épithélium thymique et son niveau de maturité. Des expériences in vitro montrent que la migration est un phénomène actif qui est lié à la sécrétion de facteurs chimiotactiques dont la nature n'est, pour le moment, pas connue. Chez les mammifères, il a été montré dans notre laboratoire (Pyke et Bach, 1979) que la migration des cellules souches dans le thymus procède d'un même mécanisme. Des fragments thymiques, des thymus fœtaux, mais aussi les surnageants de cellules épithéliales en culture, ont une activité chimiotactique qui s'exerce sur les cellules souches. Nous avons, d'autre part, pu montrer que la capacité des cellules à migrer, de même que la spécificité de cette migration, est dépendante d'un processus de maturation lié au thymus (Pyke, Papiernik et Bach, 1979). II a d'ailleurs pu être montré directement que l'incubation de cellules de foie fœtal avec le facteur thymique sérique (FTS ou thymuline) induit des modifications dans le schéma de migration des cellules souches (Pyke et Bach, 1981). Les cellules épithéliales ne sont pas les seules à intervenir dans 
ces phénomènes de migration, bien que leur rôle soit capital via la sécrétion de facteurs chimiotactiques et d'hormones. En effet, on a pu montrer que les greffes thymiques, totalement dépourvues de lymphocytes, sont moins bien colonisées par les cellules de l'hôte que des greffes de thymus non déplétées (Loor et Hagg, 1977). Ces résultats laissaient supposer un rôle complémentaire des lymphocytes thymiques dans ces phénomènes de migration. Cette hypothèse a pu être confirmée en montrant la fonction chimiotactique de surnageants produits par la stimulation des thymocytes matures (Papiernik et Laroche, 1982).

\section{Différenciation intra-thymique.}

Les cellules souches qui ont migré dans le thymus vont subir localement des influences multiples qui conduisent à la constitution de sous-populations thymocytaires, dont le niveau de maturité antigénique et fonctionnelle est variable. Une partie de ces cellules reste dans le thymus où elles peuvent avoir une longue survie (la majorité des lymphocytes médullaires) ou mourir sur place (la majorité des lymphocytes corticaux). Une petite quantité de cellules, dont le niveau de maturité est encore un sujet de controverses, quitte le thymus pour peupler les organes périphériques où elle va terminer son cycle de maturation.

La différenciation intra-thymique proprement dite se fait sous l'influence de facteurs multiples, dont nous allons analyser les composantes connues.

a. Hormones thymiques. - La notion de facteurs sécrétés par le thymus et responsables de la maturation des lymphocytes $T$, est une notion ancienne (Miller et Osoba, 1963) qui a été confirmée par la caractérisation récente de ces facteurs. Plusieurs peptides ayant des propriétés biologiques ont été isolés du thymus ou du sérum (Bach, 1979). Certains de ces peptides ont été caractérisés et séquencés. La thymosine (Goldstein et al., 1972) est en fait constituée par un ensemble de polypeptides, présents dans la fraction 5 de l'extrait thymique et dont le poids moléculaire varie entre 1000 et 15000 . La thymosine (fraction 5) est active dans plusieurs essais biologiques in vitro : modification de la réponse allogénique, en culture mixte, modification des rosettes $\mathrm{E}$ chez I'homme, modification de la réponse aux mitogènes. Elle peut reconstituer certaines fonctions immunitaires in vivo chez la souris thymectomisée, chez la souris génétiquement athymique, chez les animaux thymectomisés à l'âge adulte et chez la souris NZB. La thymosine peut modifier les antigènes de membranes des lymphocytes.

La thymopoiétine (Goldstein, 1975) a également été isolée du thymus, le test biologique initial étant l'effet sur la transmission neuro-musculaire. La thymopoiétine induit également des antigènes membranaires à la surface des lymphocytes immatures.

Le facteur thymique humoral (THF) (Trainin et al., 1975) est un peptide de petit poids moléculaire $(3000)$. Ce facteur est capable d'améliorer la compétence immunitaire in vitro de cellules d'animaux thymectomisés à la naissance (capacité à répondre en culture mixte allogénique, à tuer des cellules tumorales, et à réagir avec des lectines). L'activité du THF a également été montrée in vivo chez I'homme et chez l'animal. 


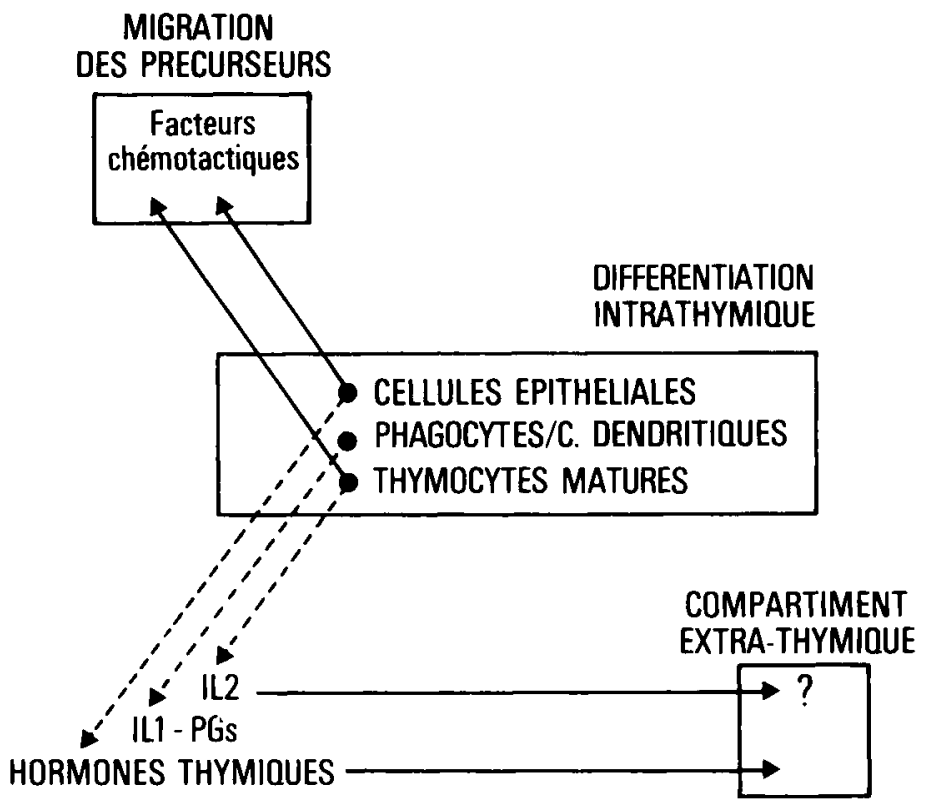

FIG. 1. - Nature et niveau d'action des facteurs sécrétés par les cellules thymiques.

La thymuline a été initialement caractérisée dans le sérum (d'où son nom initial de Facteur thymique sérique ou FTS) (Bach et Dardenne, 1973). Après la mise en évidence de zinc dans la molécule active, le FTS a été appelé thymuline (FTS-Zn). L'analyse chimique de cette hormone a permis d'en faire la séquence, et de la produire par synthèse (Pleau et al., 1977). La thymuline, comme les autres facteurs, agit sur l'expression des antigènes de différenciation. Elle est active sur la plupart des fonctions des cellules $T$ : elle augmente la cytotoxicité médiée par les cellules T chez les animaux thymectomisés; elle agit sur les cellules impliquées dans I'hypersensibilité retardée ; elle est particulièrement active sur les cellules $\mathrm{T}$ suppressives, à la fois dans des modèles in vitro et in vivo.

Les cellules épithéliales étaient supposées être à l'origine de la sécrétion des hormones thymiques. Pour la thymuline, la caractérisation de la cellule productrice était particulièrement importante puisque cette hormone avait été isolée du sérum. En utilisant un anticorps monoclonal anti-thymuline il a pu être montré récemment (Savino et al., 1982) que les cellules épithéliales thymiques sont bien à l'origine de la sécrétion de cette hormone.

Si l'on regarde l'ensemble des effets produits par les hormones thymiques, deux points d'ordre général peuvent être soulignés : 1) elles sont toutes capables d'agir sur des cellules très jeunes et d'induire la génération d'antigènes de différenciation à leur surface ; 2) en ce qui concerne l'induction ou la modification de fonctions immunitaires, la cible de ces hormones n'est pas un précurseur préthymique mais un précurseur post-thymique, plus avancé dans la séquence de dif- 
férenciation. Ces précurseurs post-thymiques sont des cellules ayant déjà reçu une influence thymique. Ces résultats étaient prévisibles compte tenu des travaux de Stutman (1978) qui montraient que les précurseurs pré-thymiques avaient l'obligation de circuler à travers le thymus pour acquérir les fonctions de cellules $T$ matures. L'injection de cellules souches à des animaux totalement dépourvus de cellules $T$ et porteurs de greffes de thymus en chambre millipore, imperméable aux cellules mais perméable aux facteurs, ne permettait pas de restaurer complètement leurs fonctions immunitaires. Par contre, la reconstitution de ces animaux était possible si la greffe de thymus était libre et colonisable par les cellules souches.

Le contact direct des cellules souches avec le micro-environnement thymique est donc un événement nécessaire à la différenciation.

b. Les cellules épithéliales. - Si les hormones thymiques sécrétées par les cellules épithéliales sont insuffisantes pour induire la maturation complète de précurseurs pré-thymiques, est-ce que le contact avec ces cellules épithéliales in vitro peut induire une telle maturation ? Autrement dit, est-ce que le micro-environnement nécessaire peut se résumer au contact entre les cellules souches et les cellules épithéliales productrices des facteurs?

Nous avons entrepris cette étude en associant des précurseurs pré-thymiques ou post-thymiques avec des cultures de cellules du réticulum thymique in vitro, contenant les cellules épithéliales. Les résultats de cette étude ont pu montrer que l'association de précurseurs pré-thymiques avec les cellules épithéliales n'induisait jamais l'apparition de fonctions immunitaires. Par contre, l'association de précurseurs post-thymiques (en l'occurrence les lymphocytes thymiques eux-mêmes) permettait I'amélioration de certaines fonctions (Papiernik et Nabarra, 1981). II semble donc que des signaux complémentaires de l'épithélium et de ses facteurs soient nécessaires à la différenciation des cellules souches dans le thymus. En particulier, un micro-environnement créé par les macrophages, les cellules interdigitées et les lymphocytes matures de la médullaire, qui sont susceptibles de sécréter des interleukines, semble nécessaire. Nous analyserons le rôle des interleukines dans le chapitre suivant.

Les cellules épithéliales sont capables de sécréter d'autres facteurs que les hormones thymiques, et en particulier chez I'homme elles peuvent produire des prostaglandines (Homo, Russo-Marie et Papiernik, 1981).

L'épithélium thymique a également focalisé l'attention par le rôle qu'on lui attribue dans l'acquisition de la restriction $\mathrm{H}-2$. II a été montré que les cellules $\mathrm{T}$ sont capables de reconnaître un antigène conventionnel lorsqu'il est associé à des antigènes du complexe majeur d'histocompatibilité ( $\mathrm{MHC}$ ) qui sont également présents sur leurs propres membranes (Zinkernagel, 1976). Les cellules souches pénétrant dans le thymus, pourraient acquérir cette restriction $\mathrm{H}-2$ au contact des cellules épithéliales qui portent à leur surface les produits du MHC (Zinkernagel et al., 1980). Cette éducation par les antigènes du "Soi 》 aurait donc un rôle très important à jouer dans les mécanismes de différenciation intra-thymique, bien que l'analyse de cette éducation chez la souris athymique vienne tempérer et moduler l'importance exclusive du rôle du thymus. 
c. Rôle des interleukines thymiques. - Les interleukines produites par le thymus semblent être un signal complémentaire important de la différenciation intra-thymique. En effet, comme nous l'avions montré pour la différenciation des précurseurs spléniques in vivo (Papiernik et Bach, 1979), les signaux ayant pour origine l'épithélium semblent avoir besoin d'un circuit d'amplification. Celui-ci peut être fourni par les interleukines qui sont produites par les cellules thymiques. Cette voie d'étude a été ouverte par l'analyse du rôle des facteurs sécrétés au cours de l'activation des lymphocytes $T$. Le premier signal, au cours de cette activation par un mitogène ou un antigène, est donné par des cellules macrophagiques qui sécrètent un médiateur, l'interleukine $1\left(I L_{1}\right)$. L' $I L_{1}$ agit en présence de l'antigène ou du mitogène, sur un lymphocyte mature qui produit un second médiateur, l'interleukine $2\left(\mathrm{IL}_{2}\right)$ qui est nécessaire à la prolifération de cellules ayant des récepteurs pour I'IL $L_{2}$ (Smith et Ruscetti, 1981).

Dans le thymus, la cellule produisant $\mathrm{I}^{\prime} \mathrm{I} \mathrm{L}_{1}$ est une cellule phagocytaire, de structure dendritique, appartenant au réticulum thymique et qui a pu être isolée en culture (Papiernik et al., 1983 ; Papiernik et Homo-Delarche, 1983). En même temps que $\mathrm{I}^{\prime} \mathrm{IL}_{1}$ (produite in vitro après stimulation par le LPS), ces cellules sécrètent des prostaglandines et, en particulier, des $\mathrm{PGE}_{2}$ qui ont un effet inhibiteur sur la prolifération des lymphocytes. Ces cellules phagocytaires du réticulum thymique (P-TR-C) ont donc un effet régulateur sur la prolifération thymocytaire en étant capables d'émettre des signaux positifs $\left(I L_{1}\right)$ et négatifs $\left(P G E_{2}\right)$ sur cette

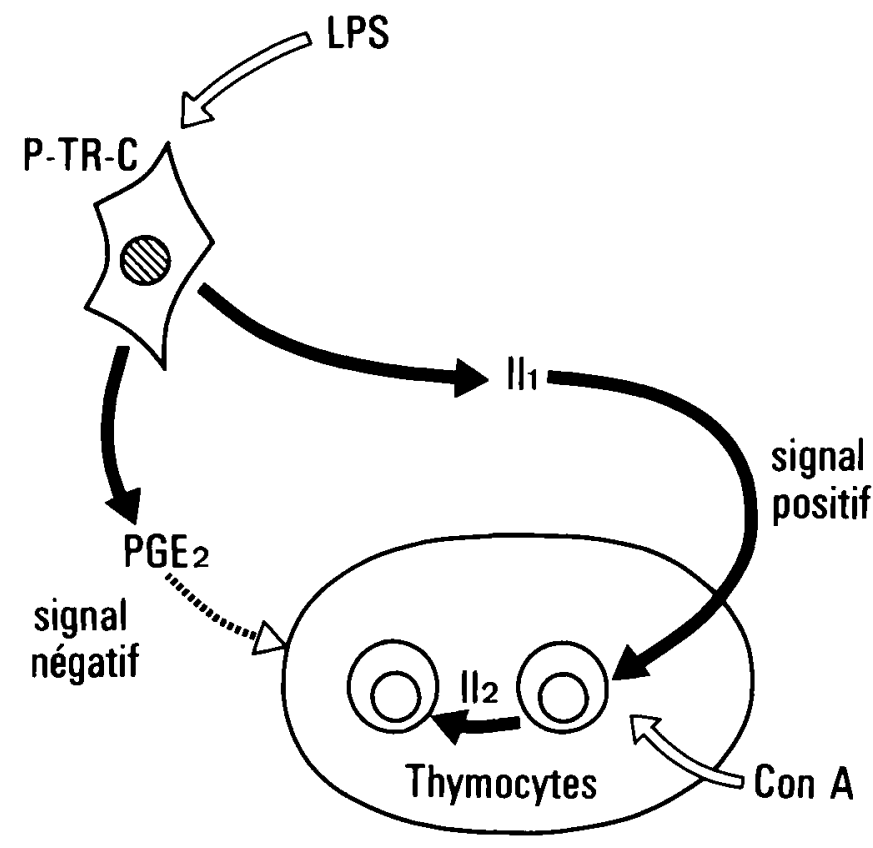

FIG. 2. - Sécrétion et niveau d'action des interleukines thymiques et des prostaglandines. 
prolifération. On peut s'interroger sur la nature du stimulus nécessaire à cette production de facteurs in vivo. On sait que les lymphocytes thymiques médullaires prolifèrent en présence de $\mathrm{P}-\mathrm{TR}-\mathrm{C}$ syngéniques. Cette stimulation syngénique est peut-être à l'origine du déclenchement de la production d'interleukines, aussi bien $d^{\prime} I L_{1}$ pour les $P-T R-C$ que $d^{\prime} I L_{2}$ par les lymphocytes matures de la médullaire thymique.

\section{Conclusion.}

Ce bref exposé des facteurs de la différenciation intra-thymique des lymphocytes $T$ permet de souligner l'extrême complexité des mécanismes en cause. Le schéma $n^{\circ} 1$ permet de résumer l'essentiel des facteurs connus. Sur la phase préliminaire de cette différenciation, qui est la migration des cellules souche vers le thymus, plusieurs types de facteurs peuvent agir : des facteurs chimiotactiques sécrétés par les cellules épithéliales, mais aussi par les lymphocytes matures de la médullaire thymique. Dans le thymus, les cellules épithéliales jouent un rôle majeur par la sécrétion des différentes hormones thymiques. Les interleukines, qui peuvent être sécrétées dans le thymus par des cellules macrophagiques et/ou dendritiques $\left(I L_{1}\right)$ et par les lymphocytes médullaires $\left(I L_{2}\right)$, sont un complément qui semble nécessaire pour amplifier les signaux de maturation épithéliaux. Les cellules macrophagiques sécrètent également des prostaglandines qui interviennent dans le circuit de régulation (tabl. 2). Certains de ces facteurs vont prolonger l'effet du thymus à la périphérie, tel la thymuline qui a été isolée du sérum et qui agit sur les phases de différenciation extra-thymique. On peut également s'interroger sur le rôle de $I^{\prime} I L_{2}$ thymique à la périphérie, au moins pendant la période péri-natale, puisque les cellules de la médullaire thymique, productrices $d^{\prime} I L_{2}$, sont les premiers lymphocytes $T$ matures de l'organisme. D'autres facteurs viendront probablement compléter ce tableau (comme l'interféron, par exemple).

Nous avons montré qu'il existe des relations étroites entre l'effet de certains facteurs comme l'I $L_{1}$ et les $P G E_{2}$. Mais l'ensemble de ces mécanismes de différenciation des lymphocytes $T$ doit être considéré comme une cascade d'événements cellulaires avec production de médiateurs dont la chronologie et les inter-relations sont mal définies. On peut s'interroger, en particulier, sur le rôle des hormones thymiques dans le déclenchement de la production d'interleukines (Palacios, 1982), sur les inter-relations qui existent entre les différentes hormones thymiques et sur la cible de leurs actions respectives, ainsi que sur les phénomènes de régulation et d'inhibition dont nous avons vu un exemple avec les prostaglandines.

Après avoir quitté le thymus, la future cellule $T$ adulte $n^{\prime}$ échappe pas à I'influence du thymus, happée par un nouveau circuit de médiateurs où les hormones thymiques et les interleukines jouent un rôle essentiel. 


\section{Références}

BACH J. F., 1979. Thymic hormones. J. Immunopharmacol, 1, 277-310.

BACH J. F., CARNAUD C., 1976. Thymic factors. Prog. Allergy., 21, 342-408.

BACH J. F., DARDENNE M., 1973. Studies on thymus products. II. Demonstration and characterization of a circulating thymic hormone. Immunology, 25, 353-366.

CEREDIG R., MAC DONALD H. R., JENKINSON E. J., 1983. Flow microfluorometric analysis of mouse thymus development in vivo and in vitro. Eur. J. Immunol., 13, 185-190.

GOLDSTEIN A. L., GUHA A., ZATZ M. M., HARDY A., WHITE A., 1972. Purification and biological activity of thymosin, a hormone of the thymus gland. Proc. nat. Acad. Sci., 69, 1800-1803.

GOLDSTEIN G., 1975. The isolation of thymopoietin (thymin). Ann. N. Y. Acad. Sci, 249, 177-183.

HOMO F., RUSSO-MARIE F., PAPIERNIK M., 1981. Prostaglandin secretion by human thymic epithelium : in vitro effect of steroid. Prostaglandins, 22, 377-387.

JOTEREAU F. V., HOUSSAINT E., LE DOUARIN N., 1980. Lymphoid stem cell homing to the early thymic primordium of the avian embryo. Eur. J. Immunol., 10, 620-627.

LOOR F., HAGG L. B., 1977. The restoration of the T lymphoid system of nude mice : lower efficiency of nonlymphoid epithelial thymus grafts. Cell. Immunol., 29, 200-204.

MILLER J. F. A. P., 1962. Effect of neonatal thymectomy on the immunological responsiveness of the mouse. Proc. roy. Soc. London B, 156, 415-428.

MILLER J. F. A. P., OSOBA D., 1963. Role of the thymus in the origin of immunological competence, p. 62. In G. WOLSTENHOLME, S. KNIGHT, Nature and origin of immunologically competent cells, Ciba Found., Study Group.

PALACIOS R., 1982. Mechanism of T cell activation : role and functional relationships of HLA-DR antigens and interleukins. Immunol. Rev., 63, 73-110.

PAPIERNIK M., 1970. Correlation of lymphocyte transformation and morphology in the human fetal thymus. Blood, 36, 470-479.

PAPIERNIK M., BACH J. F., 1979. Post natal development of T cells. I. Study of the respective contribution of thymic cellular export, thymus humoral function and $\mathrm{T}$ cell environment. J. Immunol., 123, 2311-2315.

PAPIERNIK M., HOMO-DELARCHE F., 1983. Thymic reticulum in mice. III. Phagocytic cells of the thymic reticulum in culture secrete both $\mathrm{PGE}_{2}$ and $\mathrm{IL}_{1}$ which regulate thymocyte proliferation. Eur. J. Immunol., 13, 689-692.

PAPIERNIK M., LAROCHE L., 1982. Thymic medullary lymphocytes. I. Lymphokines produced by thymic medullary lymphocytes as a second signal for intrathymic stem cell homing. Cell. Immunol., 66, 233-239.

PAPIERNIK M., NABARRA B., 1981. Thymic reticulum in mice. I. Cellular ultrastructure in vitro and functional role. Thymus, 3, 345-358.

PAPIERNIK M., NABARRA B., SAVINO W., PONTOUX C., BARBEY S., 1983. Thymic reticulum in mice. II. Culture and characterization of non epithelial phagocytic cells of the thymic reticulum. Their role in the syngeneic stimulation of thymic medullary lymphocytes. Eur. J. Immunol., 13, 147-155.

PLEAU J. M., DARDENNE M., BLOUQUIT Y., BACH J. F., 1977. Structural study of circulation thymic factor, a peptide isolated from pig serum. II. Aminoacid sequence. J. biol. Chem., 252, 8045-8047.

PYKE K. W., BACH J. F., 1979. The in vitro migration of murine fetal liver cells to thymic rudiments. Eur. J. Immunol., 9, 317-323.

PYKE K. W., BACH J. F., 1981. In vitro migration of potential hemopoietic precursors from the murine fetus. Thymus, 3, 1-7.

PYKE K. W., PAPIERNIK M., BACH J. F., 1979. Post-natal development of T cells. II. In vitro migration propertie. J. Immunol., 123, 2316-2318.

SAVINO W., DARDENNE M., PAPIERNIK M., BACH J. F., 1982. Thymic hormone containing cells : characterization and localisation of FTS in young mouse thymus studied by monoclonal antibodies. J. exp. Med., 156, 628-633.

SMITH R. A., RUSCETTI F. W., 1981. T-cell growth factor and the culture of cloned functional T cells. Adv. Immunol., 31, 137-175. 
STUTMAN O., 1978. Intra thymic and extra thymic T cell maturation. Immunol. Rev., 42, 138-184. TRAININ N., SMALL M., ZIPORI D., UMIEL T., KOOK A. I., ROTTER V., 1975. Characteristics of THF, a thymic hormone, p. 117. In D. W. VAN BEKKUM, The biological activity of thymic hormones, Kooyter sci. Publ., Rotterdam.

ZINKERNAGEL R. M., 1976. H-2 restriction of virus specific T-cell mediated effector function in vivo. II. Adoptive transfer of delayed type hypersensitivity to murine lymphocytic choriomemigitis virus is restricted by the $K$ and $D$ region of $\mathrm{H}-2 . \mathrm{J}$. exp. Med., 144. 776-787.

ZINKERNAGEL R. M., ALTHAGE A., WATERFIELD E., KINDRED B., WELSH R. M., CALLAHAN G., PINCTL P., 1980. Restriction specificities, alloreactivity and allotolerance expressed by $T$ cells from nude mice reconstituted with $\mathrm{H}-2$ compatible or incompatible thymus graft. J. exp. Med., 151, 376-399. 